\section{Peri-implant diseases: Consensus Report of the Sixth European Workshop on Periodontology}

Lindhe J, Meyle J. Peri-implant diseases: Consensus Report of the Sixth European Workshop on Periodontology. J Clin Periodontol 2008; 35 (Suppl. 8): 282-285. doi: 10.1111/j.1600-051X.2008.01283.x

\section{Abstract}

Issues related to peri-implant disease were discussed. It was observed that the most common lesions that occur, i.e. peri-implant mucositis and peri-implantitis are caused by bacteria. While the lesion of peri-implant mucositis resides in the soft tissues, periimplantitis also affects the supporting bone. Peri-implant mucositis occurs in about $80 \%$ of subjects (50\% of sites) restored with implants, and peri-implantitis in between $28 \%$ and $56 \%$ of subjects (12-40\% of sites). A number of risk indicators were identified including (i) poor oral hygiene, (ii) a history of periodontitis, (iii) diabetes and (iv) smoking. It was concluded that the treatment of peri-implant disease must include anti-infective measures. With respect to peri-implant mucositis, it appeared that non-surgical mechanical therapy caused the reduction in inflammation (bleeding on probing) but also that the adjunctive use of antimicrobial mouthrinses had a positive effect. It was agreed that the outcome of non-surgical treatment of peri-implantitis was unpredictable. The primary objective of surgical treatment in peri-implantitis is to get access to the implant surface for debridement and decontamination in order to achieve resolution of the inflammatory lesion. There was limited evidence that such treatment with the adjunctive use of systemic antibiotics could resolve a number of periimplantitis lesions. There was no evidence that so-called regenerative procedures had additional beneficial effects on treatment outcome.
Jan Lindhe ${ }^{1}$, Joerg Meyle ${ }^{2}$ on behalf of Group D of the European Workshop on Periodontology*

${ }^{1}$ Department of Periodontology, Faculty of Odontology, The Sahlgrenslea Academy at Göteborg University, Göteborg, Sweden; ${ }^{2}$ Department of Periodontology, Zentrum für Zahn-Mund-und Kieferheilkunde, JustusLiebig-Universität Giessen, Giessen, Germany

Key words: Consensus report; diagnostics; infectious diseases; non-surgical treatment; peri-implant diseases; peri-implantitis; periimplant mucositis; prevalence; risk indicators; surgical treatment

Accepted for publication 20 May 2008
Conflict of interest and source of funding statement

Group D participants declared that they had no conflict of interests.

The $6^{\text {th }}$ European Workshop on Periodontology was supported by an unrestricted educational grant from Straumann AG. The sponsor had no impact on the program or on the deliberations of the European Workshop.

\footnotetext{
* T. Berglundh, N. Claffey, H. De Bruyn, L. Heitz-Mayfield, I. Karoussis, E. Könönen, J. Lindhe, J. Meyle, A. Mombelli, S. Renvert, A. van Winkelhoff, E. Winkel, N. Zitzmann.
}

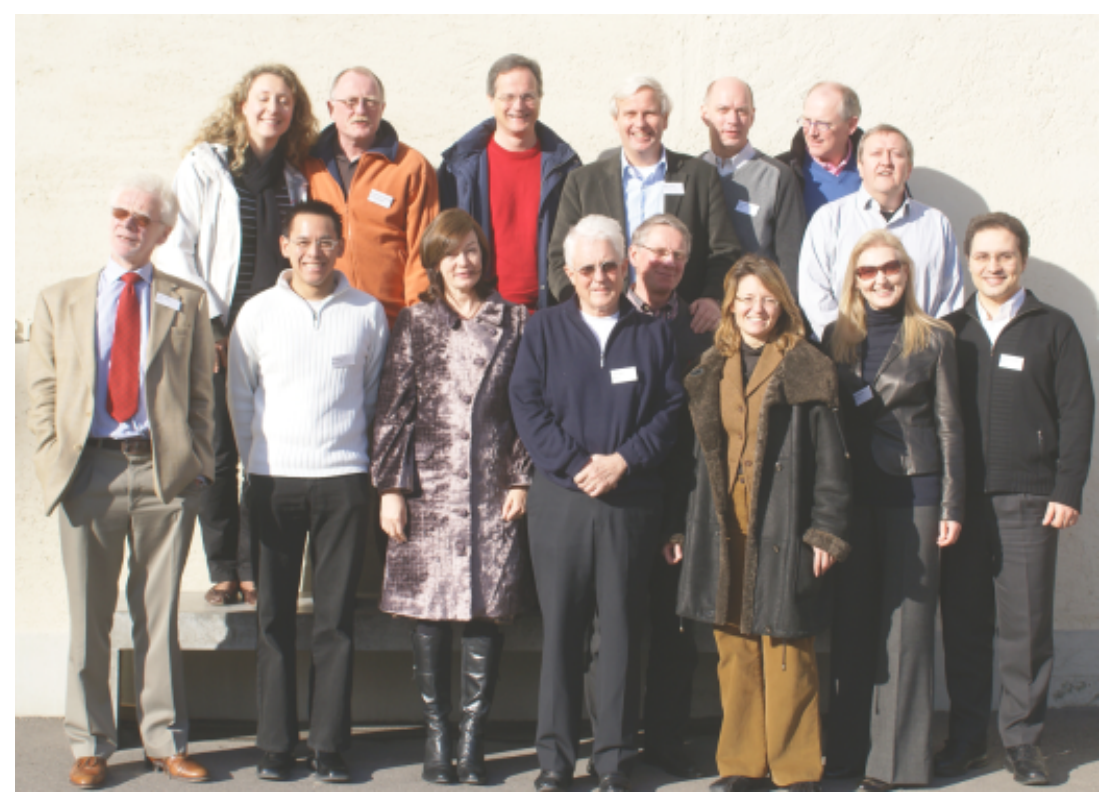

(C) 2008 The Authors Journal compilation (C) 2008 Blackwell Munksgaard 


\section{Review Papers}

Definition and prevalence of periimplant disease

\section{Zitzmann, N. U. \& Berglundh, T.}

\section{Conclusions}

Few studies provided data on the prevalence of peri-implant diseases. Crosssectional studies on implant-treated subjects are rare and data from only two study samples (662 and 216 subjects) were available. Peri-implant mucositis occurred in $80 \%$ of the subjects and in $50 \%$ of the implant sites. Peri-implantitis was identified in $28 \%$ and $\geqslant 56 \%$ of subjects and in $12 \%$ and $43 \%$ of implant sites, respectively.

Peri-implant diseases, diagnosis and risk indicators

Heitz-Mayfield, L. J. A.

Conclusions

Diagnosis

- Probing is essential for diagnosis of peri-implant diseases.

- Conventional probing using a light force $(0.25 \mathrm{~N})$ does not damage the peri-implant tissues.

- Bleeding on probing indicates the presence of inflammation in the peri-implant mucosa.

- Bleeding on probing may be used as a predictor for loss of tissue support.

- An increase in probing depth over time is associated with the loss of attachment and supporting bone.

- The probing depth, the presence of bleeding on probing and suppuration should be assessed regularly for the diagnosis of peri-implant diseases.

- Radiographs are required to evaluate supporting bone levels around implants.

- Analysis of PICF is not a clinically useful diagnostic parameter for periimplant disease.

Risks

There is evidence that the following indicators are associated with periimplant diseases:

\section{Poor oral hygiene}

An OR of 14.3 (95\% CI: 2.0-4.1) was shown in two studies.

\section{History of periodontitis}

Four systematic reviews and 10 of 11 studies (comparing patients with a history of periodontitis with patients without a history of periodontitis) show an increased risk for peri-implant disease.

\section{Cigarette smoking}

A systematic review reported five retrospective and one prospective study showing an association of smoking and peri-implantitis. Twelve of thirteen studies showed a significant increase in marginal bone loss in smokers compared with non-smokers.

There is limited evidence that the following indicators are associated with peri-implant diseases:

1. Diabetes with poor metabolic control

2. Alcohol consumption

There is conflicting and limited evidence for an association with periimplant diseases:

1. Genetic traits

- One author showed a significant association with peri-implantitis two others not.

- Three studies provided evidence for a synergism between genetic traits and smoking.

\section{Implant surface}

Two studies with implants with different surfaces within the same patient demonstrated that rough was worse than smooth, moderately rough similar to smooth.

Where clinical assessment indicates the presence of peri-implant disease, a radiograph should be taken in order to determine the extent of bone loss.

Non-surgical treatment of peri-implant mucositis and peri-implantitis: A literature review

Renvert, S., Roos-Jansåker, A. M. \& Claffey, $\mathbf{N}$.

\section{Conclusions}

- It was observed that mechanical non-surgical therapy could be effective in the treatment of peri-implant mucositis lesions. Furthermore, the adjunctive use of antimicrobial mouthrinses enhanced the outcome of mechanical therapy of such mucositis lesions.

- In peri-implantitis lesions, non-surgical therapy was not found to be effective. Adjunctive chlorhexidine application had only limited effects on clinical and microbiological parameters. However, adjunctive local or systemic antibiotics were shown to reduce bleeding on probing and probing depths.

- Minor beneficial effects of laser therapy on peri-implantitis have been shown; this approach needs to be further evaluated.

Surgical treatment of peri-implantitis

Clarke, E., Polyzois, I., Renvert, S. \& Claffey, $\mathbf{N}$.

\section{Conclusions}

\section{Animal studies}

- Open debridement including surface decontamination was more effective in the treatment of peri-implantitis than closed debridement.

- Open debridement including surface decontamination resolved periimplantitis, promoted bone fill and could result in re-osseointegration. Re-osseointegration was more pronounced on rougher than on smooth implant surfaces.

- No single method of surface decontamination was found to be superior.

- The adjunctive use of graft material, with or without membranes resulted in varying amounts of bone fill and re-osseointegration. However, this effect appeared to be influenced by the size and morphology of the periimplant defect.

\section{Human studies}

- Access surgery combined with implant surface decontamination for treatment of peri-implantitis has scarcely been investigated. The only study available also included the use of systemic antibiotics and found that resolution occurred in about $60 \%$ of the treated sites.

- No single method of surface decontamination (chemical agents, air abrasives and lasers) was found to be superior. 
- So far, it is not known if the adjunctive use of systemic antibiotics in surgical therapy of peri-implantitis is required.

- Regenerative procedures such as bone graft techniques with or without the use of barrier membranes resulted in various degrees of success. However, it must be stressed that such techniques do not address disease resolution but rather merely attempt to fill the osseous defect.

\section{General Conclusions of Group D}

The issues presented in the following relate to osseointegrated implants in function.

\section{Definitions}

Peri-implant mucositis and peri-implantitis are infectious diseases.

Peri-implant mucositis describes an inflammatory lesion that resides in the mucosa, while peri-implantitis also affects the supporting bone.

\section{Diagnosis}

Peri-implant mucositis: Peri-implant mucositis may be identified clinically by redness and swelling of the soft tissue, but bleeding on probing is currently recognized as the important feature.

Peri-implantitis: In peri-implantitis, the mucosal lesion is often associated with suppuration and deepened pockets, but always accompanied by loss of supporting marginal bone.

\section{What is the prevalence of peri-implant diseases?}

There are limited data available related to the prevalence of peri-implant disease. Thus, the search could identify only three cross-sectional reports including two subject samples presenting information on only one implant system. Six hundred and sixty-two subjects (3413 implants) were included in one publication and 216 (987 implants) in another one.

Peri-implant mucositis: In the review, the diagnosis 'peri-implant mucositis' was based on the sign 'bleeding upon probing' without loss of supporting bone. The authors reported that approximately $80 \%$ of the subjects and $50 \%$ of the implant sites exhibited peri-implant mucositis.
Peri-implantitis: In the two reports, the prevalence of peri-implantitis identified by bleeding upon probing and bone loss (after 1 year in function) and representing only one implant system - was identified in between $28 \%$ and at least $56 \%$ of subjects and in $12 \%$ and $43 \%$ of implant sites.

\section{Risk for peri-implant disease}

In the literature, most of the studies report implant loss, early and late losses, without referring to peri-implant disease.

In the current review, the term 'risk' was used in the context of factors found to be associated with peri-implant disease. The following factors were identified: poor oral hygiene, a history of periodontitis, diabetes, smoking, alcohol consumption and genetic traits. The best evidence is with poor oral hygiene, with a history of periodontitis and with cigarette smoking, while the association with other factors is less well established.

\section{Treatment}

\section{Common sense}

The success of treatment outcome must include parameters that describe resolution of inflammation and preservation of the supporting bone. Because periimplant diseases are caused by bacteria, treatment must include anti-infective measures.

\section{Non-surgical treatment}

Limited evidence from case series indicates that there may be a beneficial effect of non-surgical therapy.

Peri-implant mucositis: It has not been documented that mechanical therapy alone is effective in reducing bleeding upon probing. In two prospective studies it was demonstrated, that the adjunctive use of antimicrobial mouthrinses reduced the percentage of bleeding sites.

Peri-implantitis: Case control studies suggested that a non-surgical approach to peri-implantitis could be beneficial.

In addition, four comparative clinical trials documented that the adjunctive use of local antibiotics reduced the number of bleeding sites and their probing depths. It should be observed that the studies included in the review demonstrated varying degrees of disease resolution.

Conclusions: Based on evidence, it seems that the outcome of non-surgical therapy is unpredictable.

\section{Surgical treatment}

The primary objective of surgical treatment in peri-implantitis lesions is to get access to the implant surface for debridement and decontamination in order to achieve resolution of the inflammatory lesion. Animal studies have documented that this can occur.

Peri-implantitis: Several studies on periodontitis have reported the longterm success of various treatment modalities, including anti-infective measures.

Similar long-term studies on the treatment of peri-implantitis were not available.

Limited evidence from one case series indicates, that surgical treatment including implant surface decontamination and systemic antibiotics resolved a number of lesions. During the 5 years of follow-up, however, seven implants in four patients were lost and four implants exhibited disease progression. In six sites, new bone formation could be observed.

There is no evidence, that so-called 'regenerative procedures' [bone grafts/ substitutes, guided bone regeneration (GBR)], performed in implant sites with bone craters, have additional beneficial effects on treatment outcome.

\section{Clinical implications}

- The patient should be informed that peri-implant tissues respond to plaque accumulation in a way similar to that of periodontal tissues and that disease may develop in the tissues around the implants jeopardizing their longevity.

- Poor oral hygiene resulting in plaque accumulation leads to periimplant disease.

- Bleeding on probing of the periimplant tissues is a sign of inflammation in the continued presence of which loss of supporting bone may occur.

- The clinician should be aware that suppuration in an implant site is often a sign of peri-implantitis.

- The clinician must perform probing in order to monitor the peri-implant conditions and diagnose periimplant disease. 
- Baseline probing measurements should ideally be recorded at approximately the time of placement of the suprastructure.

- At least, annual monitoring of the peri-implant probing depths and the presence of bleeding on probing and suppuration must be performed to allow comparisons with the baseline recordings and to allow early diagnosis of peri-implant disease.

- It should be observed that the profile of the implant and the contour of the reconstruction might hinder probing at four surfaces per implant. In such a case, at least one surface must be identified, where proper probing measurements can be performed.

- Probing using a conventional periodontal probe damages neither the mucosal attachment nor the implant.

- Baseline radiographs should be taken approximately at the time of placement of the suprastructure to establish the level of supporting bone.

- When clinical signs suggest the presence of peri-implantitis, the clinician is advised to take a radiograph of the site to confirm the diagnosis.

- Mobility of an implant indicates the complete lack of osseointegration and calls for its removal.

- Patients with a history of periodontitis must be informed that they are more at risk for peri-implant disease.

- It is imperative that periodontal disease is treated before implant placement and that the patient receives proper periodontal maintenance care.

- Smokers should be informed that they are more at risk for periimplant disease.

- The clinician should inform the diabetic patient that she/he may have an increased risk for peri-implantitis.

\section{Clinical Relevance}

Based on the current scientific evidence, as outlined in the original
- Bacterial deposits must be removed in the treatment of peri-implant mucositis and peri-implantitis.

- The clinician should be aware that peri-implantitis is difficult to treat and the outcomes may not be predictable.

- If non-surgical mechanical therapy does not resolve the lesion, adjunctive antimicrobials and access flap surgery are recommended.

- In order to restore the hard-tissue defect, reconstructive surgery may be considered.

\section{Implications for future research}

- For the purpose of providing sufficient information on the prevalence of peri-implant disease, an epidemiological approach is recommended. Using a cross-sectional design and a study sample with an appropriate size, clinical and radiographic data should be collected. Ideally, the subjects should be recruited from private or public dental clinics, rather than university clinics and, hence, provide information on the 'effectiveness' rather than 'efficacy' in implant therapy.

- The extent as well as the severity (amount of bone loss) of the lesion needs to be described, i.e. the proportion of affected implants in each subject of the sample.

- Longitudinal studies on large subject samples must be performed in order to confirm that factors associated with peri-implant disease (e.g. diabetes, smoking, genetic traits, etc.) are indeed risk factors or indicators.

papers, this consensus report is of crucial importance for clinical prac-
- There is a need to determine whether antimicrobials used in periodontal therapy are also effective in the treatment of peri-implant diseases, and to what extent initial improvements are sustained over the long term.

- A number of issues associated with surgical treatment of peri-implantitis and its sequelae require further investigation, including methods for implant surface decontamination, use of antibiotics and regenerative procedures.

- Future studies must investigate the influence of defect characteristics or patient-related factors on treatment outcome.

\section{References}

Clarke, E., Polyzois, I., Renvert, S. \& Claffey, N. (2008) Surgical treatment of peri-implantitis. Journal of Clinical Periodontology 35 (Suppl. 8), 316-332.

Heitz-Mayfield, L. J. A. (2008) Peri-implant diseases, diagnosis and risk indicators. Journal of Clinical Periodontology 35 (Suppl. 8), 292-304.

Renvert, S., Roos-Jansåker, A.-M. \& Claffey, N. (2008) Non-surgical treatment of periimplant mucositis and peri-implantitis: a literature review. Journal of Clinical Periodontology 35 (Suppl. 8), 305-315.

Zitzmann, N. U. \& Berglundh, T. (2008) Definition and prevalence of peri-implant disease. Journal of Clinical Periodontology 35 (Suppl. 8), 286-291.

Address:

Joerg Meyle

Department of Periodontology

Zentrum für Zahn-Mund-und Kieferheilkunode Justus-Liebig-Universität Giessen

Giessen

Germany

E-mail: joergy.meyle@dentist.med.uni_gissen.de

tice in the diagnosis and treatment of peri-implant diseases. 